\title{
Detection of Different Oxidation States of Individual Manganese Porphyrins During their Reaction with Oxygen at a Solid/Liquid Interface
}

Duncan den Boer ${ }^{1,2 \dagger}$, Min $\mathrm{Li}^{3,4 \dagger}$, Thomas Habets ${ }^{1}$, Patrizia Iavicoli ${ }^{5}$, Alan E. Rowan ${ }^{1}$, Roeland J. M. Nolte ${ }^{1}$, Sylvia Speller, ${ }^{1,6}$, David B. Amabilino ${ }^{5 *}$, Steven De Feyter ${ }^{3^{*}}$ and Johannes A. A. W. Elemans ${ }^{1 *}$

1 Radboud University Nijmegen, Institute for Molecules and Materials (IMM), Heyendaalseweg 135, 6525 AJ Nijmegen, The Netherlands

2 Current address: Department of Chemistry, Massachusetts Institute of Technology, 77 Massachusetts Avenue, Cambridge, Massachusetts 02139, USA

3 KU Leuven - University of Leuven, Division of Molecular Imaging and Photonics, Celestijnenlaan 200-F, 3001 Leuven, Belgium

4 Current address: CAS Key Laboratory for Biomedical Effects of Nanomaterials and Nanosafety, Institute of High Energy Physics, Chinese Academy of Sciences, Beijing, 100049, China

5 Institut de Ciència de Materials de Barcelona (ICMAB-CSIC), Campus Universitari, 08193 Bellaterra, Catalonia, Spain

6 Current address: Institut für Physik, University of Rostock, Rostock, Germany

$†$ These authors contributed equally.

*To whom correspondence should be addressed; E-mail: J.Elemans@science.ru.nl (J.A.A.W.E.); Steven.DeFeyter@chem.kuleuven.be (S.D.F.); amabilino@icmab.es (D.B.A.). 
Manganese porphyrins have been extensively investigated as model systems for the natural enzyme cytochrome P450 and as synthetic oxidation catalysts. We report here single molecule studies of the multistep reaction of manganese porphyrins with molecular oxygen at a solid/liquid interface, employing a scanning tunneling microscope (STM) under environmental control. The high lateral resolution of the STM, in combination with its sensitivity for subtle differences in electronic properties of molecules, allowed the detection of at least four distinct reaction species. Real-space and real-time imaging of reaction dynamics enabled the observation of active sites, immobile on the experimental timescale. The conversions between the different species could be tuned by the composition of the atmosphere (argon, air or oxygen) and the surface bias voltage. By means of extensive comparison of the results to those obtained by analogous solution-based chemistry, we assigned the observed species to the starting compound, reaction intermediates, and products.

Cytochrome P450, an abundant enzyme in the animal, plant and microbial kingdoms, acts as a mono-oxygenase in various detoxification and biosynthesis pathways ${ }^{1}$. Its active site contains an $\mathrm{Fe}$ (III)-heme complex, which can activate molecular oxygen for the oxidation of a variety of organic substrates. Taking this efficient natural catalyst as a blueprint, many artificial systems containing $\mathrm{Mn}$ (III) or $\mathrm{Fe}$ (III) porphyrins have been developed ${ }^{2}$ and used in catalytic oxidation reactions ${ }^{3}$. Numerous studies have been devoted to elucidating the role of manganese porphyrin in the complex oxygen transfer mechanisms between oxidant and substrate $^{4-7}$. The majority of these studies use ensemble techniques in solution, such as electrochemistry and absorption, electron paramagnetic resonance and nuclear magnetic resonance spectroscopy. The scanning tunneling microscope (STM) has proven to be a promising tool to study reactions at the molecular level ${ }^{8-12}$, and initial experiments have been reported in which the reactive properties of metal porphyrins were studied on a surface at the single molecule level by STM in ultrahigh vacuum (UHV) ${ }^{13-17}$ and under ambient conditions $^{18,19}$.

Here we report detailed STM studies of the complex redox chemistry of reactions between meso-5,10,15,20-Tetrakis[4-(R,R,R,R)-2-N-octadecylamidoethyloxyphenyl]porphyrin manganese(III) chloride (Mn1Cl, Fig. 2a) and different oxygen donors at a solid/liquid interface. Although the dynamic nature of the molecules at this interface complicates the use of techniques that are typically being used under UHV conditions, such as X-ray 
Photoelectron Spectroscopy (XPS), STM can provide extremely high spatial resolution and insight into changes in the electronic properties of molecules with a liquid medium over them. These liquid conditions make the system far more comparable with processes taking place in biological systems than the UHV conditions, where solvent-mediated factors such as the diffusion and concentration of reactants are fully absent. It is in principle possible with STM to monitor single molecules at the highest detail while they are involved in multistep chemical reactions with STM, and to image and identify reactants, intermediates and products in the most direct way, i.e. by imaging. This approach allows the study of reaction dynamics in realspace and real-time, which may provide unique information about reaction mechanisms that remains hidden in ensemble measurements at the macroscopic scale. STM may reveal variations in reactivity of single molecules, the relation of these variations to molecular adsorption geometry, and cooperativity effects at the nanometre scale.

An overview of the possible reaction pathways involving manganese porphyrins and oxygen, based on the literature $\mathrm{e}^{1-3,18,20-29}$ and the observations described here, is depicted in Figure 1. A reduction of the manganese centre of the porphyrin from $\mathrm{Mn}(\mathrm{III})$ to $\mathrm{Mn}$ (II) with loss of the counterion $^{20}$ is a requirement for the binding of an oxygen molecule to give a dioxygen adduct ${ }^{21}$. Subsequently, the oxygen-oxygen bond of the resulting species can be cleaved following two possible pathways: (i) a reaction with $\mathrm{Mn}$ (II) to form two $\mathrm{Mn}(\mathrm{IV}$ )-oxo complexes $^{18,22,23}$; (ii) a reaction with an electron and two protons to generate water and a $[\mathrm{Mn}(\mathrm{V})-\mathrm{oxo}]^{+}$species $^{1-3}$. The Mn(IV)-oxo species is active in the epoxidation of alkenes ${ }^{3,24}$, and in the absence of such substrates it can connect to an additional $\mathrm{Mn}$ (II) porphyrin to give a $\mu$-oxo bridged $\mathrm{Mn}$ (III) dimer ${ }^{25-28}$, or to an $\mathrm{Mn}$ (III) porphyrin to give a mixed valence $[\mathrm{Mn}(\mathrm{IV})-\mathrm{O}-\mathrm{Mn}(\mathrm{III})]^{+}$dimer $^{29}$. As an alternative to molecular oxygen, a single oxygen donor such as pentafluoroiodosylbenzene (PFIB) can react directly with an $\mathrm{Mn}$ (III) porphyrin to generate a $[\mathrm{Mn}(\mathrm{V}) \text {-oxo }]^{+}$species, which can further react with an $\mathrm{Mn}(\mathrm{III})$ porphyrin to form a $[\mathrm{Mn}(\mathrm{IV})-\mathrm{O}-\mathrm{Mn}(\mathrm{IV})]^{2+}$ dimer $^{1,3}$. The aim of this research is the detection of the individual species depicted in Scheme 1 at the single molecule scale by STM, in order to provide realtime and real-space mechanistic information about reaction dynamics at the chemically and biologically relevant solid/liquid interface. With our approach elucidation of the full structural details of the reaction products is beyond the sensitivity level, since the sophisticated spectroscopy that is usually required for the ensemble characterisation of such species in solution is not applicable at the solid/liquid interface at the level of individual molecules. Our intention is to apply STM as a single molecule technique complementary to the conventional 
ensemble techniques, in order to detect different states and infer them to be associated with different species by logical analogy with the solution chemistry.

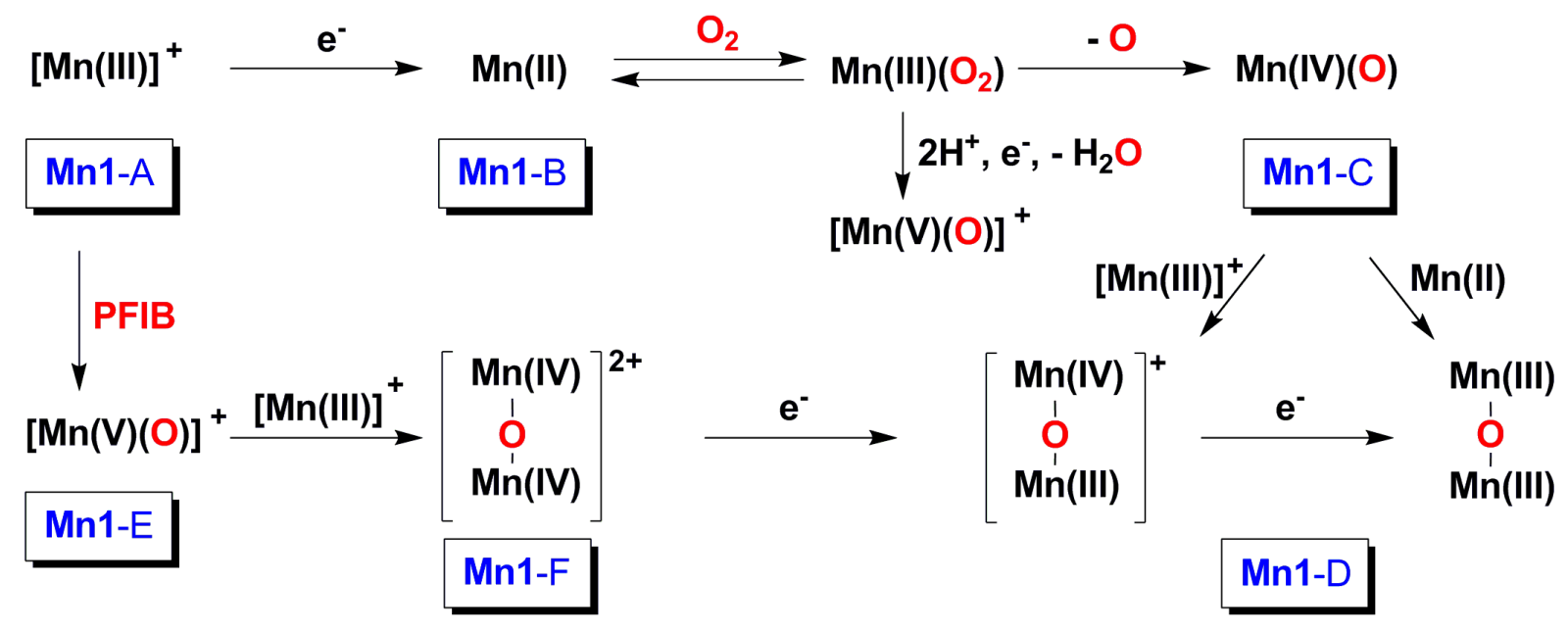

Figure 1 | Possible pathways and states of the manganese centre of Mnporphyrins during their reaction with $\mathrm{O}_{2}$ or with the single oxygen donor pentafluoroiodosylbenzene (PFIB); the STM topographies linked to the various states in this paper are depicted in blue. Before it can react with $\mathrm{O}_{2}$ the manganese centre of the native Mn-porphyrin (Mn1-A) must first be reduced to $\mathrm{Mn}$ (II) (Mn1-B) with the loss of the chloride counter ion. After reaction of this species with $\mathrm{O}_{2}$ a $\mathrm{Mn}(\mathrm{III})$-dioxo complex is formed, which can either react with two protons to generate a $\mathrm{Mn}(\mathrm{V})$-oxo species and a water molecule, or give a $\mathrm{Mn}(\mathrm{IV})$-oxo species (Mn1-C) after homolytic dissociation of the oxygen-oxygen bond. This species can react with either a $\mathrm{Mn}(\mathrm{III})$ or a $\mathrm{Mn}$ (II)-porphyrin to yield a mixed valence $\mathrm{Mn}(\mathrm{IV})-\mathrm{O}$ $\mathrm{Mn}$ (III) or a Mn(III)-Mn(III) $\mu$-oxo-porphyrin dimer, respectively. Based on STM topography no discrimination could be made between these two complexes, therefore the assignment Mn1-D is placed in between them. When Mn1-A reacts with PFIB it generates a Mn(V)-oxo porphyrin (Mn1-E), which can further react with a $\mathrm{Mn}$ (III) porphyrin to give a Mn(IV)-Mn(IV) $\mu$-oxo-porphyrin dimer (Mn1-F).

In a first experiment we deposited a droplet of a $\sim 10^{-5} \mathrm{M}$ solution of $\mathbf{M n 1 C l}$ in 1-octanoic acid at the basal plane of a freshly cleaved highly oriented pyrolytic graphite (HOPG) sample under ambient conditions. Related alkyl-functionalised free base porphyrin molecules are known to form well-ordered monolayers on the same substrate ${ }^{30,31}$. STM revealed the 
instantaneous self-assembly of the molecules of $\mathbf{M n 1 C l}$ in extended lamellar arrays, in which the porphyrin planes are adsorbed parallel to the surface and the alkyl chains interdigitated (Fig. 2B). The orientation of the lamellae and the alkyl chains with respect to the main crystallographic directions of the graphite surface is identical to that observed for the corresponding free base derivative. ${ }^{30}$ When the surface was scanned under ambient conditions at a negative bias voltage of $-800 \mathrm{mV}$ and a tunneling current setpoint of $10 \mathrm{pA}$, typically within tens of minutes four distinct topographical Mn1-related states became apparent, which we label Mn1-A, Mn1-B, Mn1-C and Mn1-D (Figs 2C and 2D). These states were stable and remained present for many minutes up to several hours. The stability of the monolayer at phase boundaries and defects indicated that molecular desorption and readsorption processes were very infrequent at the timescale of the experiment (Supplementary Fig. S8). Neither the tip material (Pt/Ir), nor the solvent were a determinant in the formation of the states, as experiments with a gold tip or at a HOPG/1-phenyloctane interface revealed similar topographical features (Supplementary Figs S9 and S10). In all STM experiments Mn1-A was the most abundant state and at the start of the measurement generally the only one present, therefore it is expected to correspond to the native compound $\mathbf{M n}(\mathbf{I I I}) \mathbf{1 C l}$.
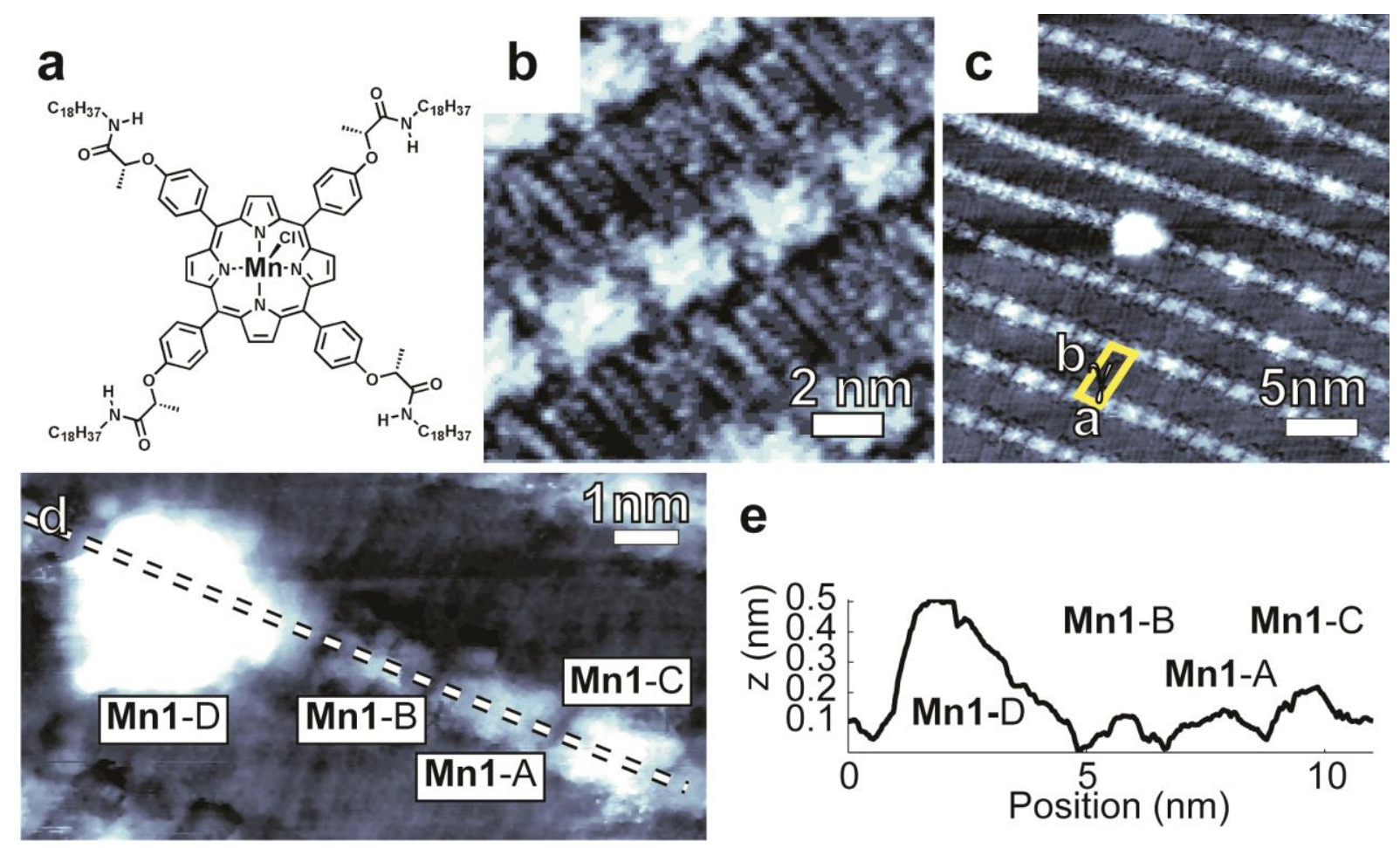

Figure 2 | Appearance of Mn-porphyrins at the solid/liquid interface. A, Molecular structure of $\mathbf{M n} 1 \mathrm{Cl}$. B, STM topography image of $\mathbf{M n 1 C l}$ at the HOPG/1octanoic acid interface under ambient conditions, directly after monolayer formation. 
$V_{\text {bias }}=-800 \mathrm{mV}, I_{\text {set }}=50 \mathrm{pA}$. C, Idem, after 8 hours. The unit cell is drawn in: $\mathrm{a}=1.9$ $\pm 0.15 \mathrm{~nm}, \mathrm{~b}=4.0 \pm 0.1 \mathrm{~nm}$ and $\gamma=80 \pm 3^{\circ}$. $V_{\text {bias }}=-800 \mathrm{mV}$, $I_{\text {set }}=10 \mathrm{pA}$. D, Magnification of image C. The distinct states of Mn1 are indicated. E, Cross-section corresponding to the white dashed line in image $\mathbf{D}$.

Given the known ability of manganese porphyrins to react with oxygen, a possible correlation of the emergence of the distinct topographical states of $\mathbf{M n 1 C l}$ and the presence of $\mathrm{O}_{2}$ was checked by performing STM measurements under different controlled atmospheres. For this purpose, the microscope was covered with a glass bell-jar, and in two separate experiments the emergence of the states was monitored in time in either an argon or an oxygen atmosphere (Figs 3A and 3B). The most striking difference between the two experiments was the increase in relative surface coverage of Mn1-C: after $4.5 \mathrm{~h}$ in an argon atmosphere, its relative coverage was about $3 \%$, while after the same time in an oxygen atmosphere it amounted to more than $20 \%$ of the surface-bound molecules. The scanning by the STM tip was not the major cause of the appearance of this state, since previously unscanned areas at remote locations ( $>1 \mu \mathrm{m}$ away) revealed a similar abundance to an area that was scanned for hours (Supplementary Figs S11 and S12). We therefore conclude that the formation of Mn1-C is directly related to the presence of oxygen, which is remarkable, since UV-visible absorption spectroscopy studies revealed that in 1-octanoic acid solution Mn1 is inert to this gas. This suggests the presence of a surface effect. 


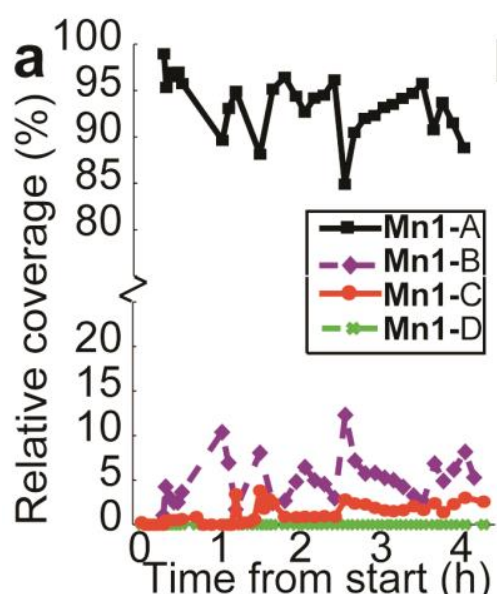

b $\begin{array}{r}100 \\ 95\end{array}$
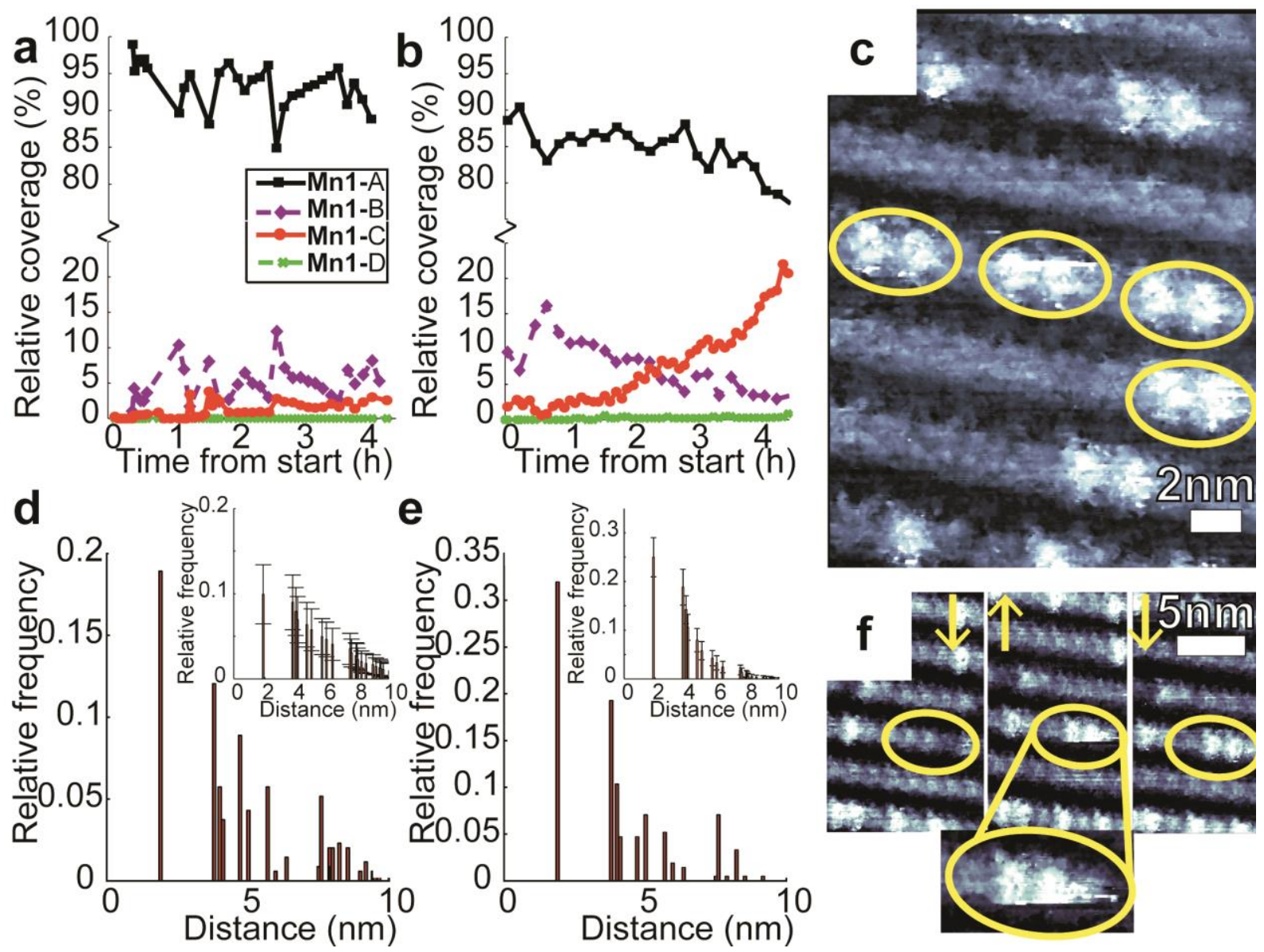

Figure 3 | Mn-porphyrin states at the solid/liquid interface under different

atmospheres. A, Relative coverages of Mn1-A, Mn1-B, Mn1-C and Mn1-D as function of time from the start of the STM measurement under argon. B, idem, under oxygen. In both cases 300-500 molecules were monitored. $V_{\text {bias }}=-800 \mathrm{mV}, I_{\text {set }}=5$ pA. C, STM topography image of Mn1, pairs of Mn1-C at adjacent locations in the monolayer are indicated by yellow ellipses. $V_{\text {bias }}=-800 \mathrm{mV}, I_{\text {set }}=5 \mathrm{pA}$. D, Nearest neighbour distributions for Mn1-C species in the STM experiment with oxygen with relative coverages between $0-8 \%\left(N_{\exp }=349, t=0.5\right.$ to $\left.2 \mathrm{~h}\right)$. Inset: corresponding nearest neighbor distribution of simulation datasets with randomly distributed species ( $\mathrm{N}_{\mathrm{sim}}=1000$ sets, error bars indicate S.D.). E, Idem, for relative coverages between $10-16 \%\left(N_{\text {exp }}=213, t=2.5\right.$ to $\left.4 \mathrm{~h}\right)$. Inset: corresponding simulation with randomly distributed species ( $\mathrm{N}_{\mathrm{sim}}=1000$ sets, error bars indicate S.D.). F, STM topography images of the formation of adjacent Mn1-C complexes between two scan lines; $V_{\text {bias }}$ $=-800 \mathrm{mV}, I_{\mathrm{set}}=5 \mathrm{pA}$, time between scan lines $380 \mathrm{~ms}$, time between two images 3 minutes. The slow scan direction is indicated by the arrows. 
State Mn1-C may be assigned to an $\mathbf{M n}(\mathrm{III}) \mathbf{1}\left(\mathrm{O}_{2}\right)$ complex, but such a species has been reported to be thermally very unstable in solution. ${ }^{32-35}$ STM measurements indicated that Mn1-C was generally stable for at least an hour. Furthermore, inspection of many STM images indicated that the molecules of Mn1-C were distributed non-randomly over the surface. In particular, there seemed to be a preference for the emergence of state Mn1-C at adjacent locations in the lamellae (Fig. 3C). When experimentally obtained nearest neighbor distribution histograms of Mn1-C (at $<8 \%$ surface coverages to minimise the effect of occupation statistics) were compared to a simulated dataset assuming a random distribution in the assembly, the experimental dataset revealed that the relative occurrence at the closest possible distance between two molecules (at $1.9 \mathrm{~nm}$ ) is about twice as high as the corresponding peak in the simulation dataset (Fig. 3D and Supplementary Material). Such a distribution points at a correlated process, and might be explained by a similar preferential pair-wise generation of state Mn1-C (illustrated in Fig. 1) as was previously observed for the reaction of oxygen with another type of Mn porphyrins adsorbed on a $\mathrm{Au}(111)$ surface $^{18}$. At higher surface coverages of Mn1-C of 10-16\%, this correlation appears not so pronounced when compared to the corresponding simulation (Fig. 3E), although in that case occupation statistics can mask such an effect. The apparent correlation in the formation of these species can be explained by reasoning that Mn1-C corresponds to an Mn(IV)-oxo porphyrin complex, which can form as a result of a homolytic splitting of the oxygen-oxygen bond ${ }^{18,22,36}$. In that case the second oxygen atom can connect to a neighboring manganese porphyrin on the surface, whose effective molarity is extremely high, ${ }^{37}$ to generate another $\mathrm{Mn}(\mathrm{IV})$-oxo species. A related proximity effect in which two adjacent catalytic metal centres are involved in binding and splitting of molecular oxygen has been recently imaged in an STM study in which di-iron complexes were studied under UHV conditions ${ }^{36}$. In the present case it is noteworthy that the process takes place in a fluid medium.

An additional indication for the correlation in the formation of Mn1-C came from the observation in real-time of single molecule formation events by STM. We define such an event as the appearance of an Mn1-C species at a location in the monolayer at which in the previously scanned STM image the porphyrin molecule existed in a different state. In a set of 115 unique events of this type occurring in one STM measurement series, 28 of the Mn1-C species (24\%) had an Mn1-C species that also formed at the same time at the nearest neighbour site. This high fraction cannot be explained by a generation as randomly distributed single events, as in that case a much lower fraction of formation of Mn1-C at nearest neighbor 
sites is expected (a simulation for the generation of pairwise formation of Mn1-C shows that this fraction is around 3-4\%, see Supplementary Fig. S3). In several cases, pairwise formation events could be observed in between two scan lines (time interval $380 \mathrm{~ms}$ ) in the STM images (Fig. 3F and Supplementary Figs S6 and S7).

It is as yet unclear how the transfer of the second oxygen atom to a neighbouring Mn1 porphyrin takes place. A mechanism involving a homolytic dissociation of an $\mathrm{Mn}-\mathrm{O}-\mathrm{O}-\mathrm{Mn}$ intermediate $^{22}$ would require a 'flipping up' of two adjacent porphyrin planes to simultaneously bind an oxygen molecule, but we have not observed such an intermediate in our STM measurements. An alternative is the surface-mediated diffusion of atomic oxygen, i.e. via the adsorbed porphyrin planes, to a neighbouring Mn1 molecule. However, the observation that $76 \%$ of the formation events do not yield species Mn1-C at nearest neighbour sites indicates strongly that the majority of the oxygen atoms react in a different way, for example by diffusing away from the surface and reacting with molecules of the solvent or molecules dissolved therein.

A reaction of $\mathbf{M n 1 C l}$ with a single oxygen donor should generate oxidised manganese porphyrin species in a non-correlated fashion, and lead to a random distribution in the monolayer and a lower peak at the adjacent monolayer location in the nearest neighbor distribution than for the experiments with molecular oxygen. To test our hypothesis, PFIB was added to a freshly assembled monolayer of $\mathbf{M n 1 C l}$ at the HOPG/1-octanoic acid interface. It was expected that this reagent would generate a $\left[\mathbf{M n}(\mathbf{V}) \mathbf{1}-\right.$ oxo $^{+}{ }^{+}$species $^{1,3}($ Fig. 1). Immediately after its addition STM images revealed the formation of a considerable amount of two topographical states, which we label Mn1-E and Mn1-F (Fig. 4A). While Mn1-F strongly resembled the signature of Mn1-D in the experiment with oxygen, Mn1-E has a topography characterised by bright spikes that have a similar apparent height as the topography of Mn1-C (Fig. 4B and Supplementary Fig. S13). Its relative coverage rapidly increased to $13 \%$ within 45 minutes. Considering the results of numerous oxidation studies with manganese porphyrins, we assign Mn1-E as a $[\mathbf{M n}(\mathbf{V}) \mathbf{1}-o x o]^{+}$species (see Fig. 1). Its appearance as spikes, when compared to Mn1-C, might be attributed to a bound oxygen atom possibly less stable than the other case, or to the fact that the proposed species is charged, with its chloride counter ion being non-coordinated but still present in the outer-coordination shell of the molecule and mobile under the action of the STM tip. When the nearest neighbor distribution histogram of molecules in state Mn1-E (Fig 4C) is compared to that of molecules in state Mn1-C in the experiment with oxygen, at a similar surface coverage (Fig. 3E), it turns 
out that in the case of the oxidation reaction using PFIB the peak corresponding to adjacent monolayer locations is lower and in fact in very good agreement with a simulation assuming the formation of Mn1-E with a random spatial distribution on the surface (cf. Supplementary Fig. S2).
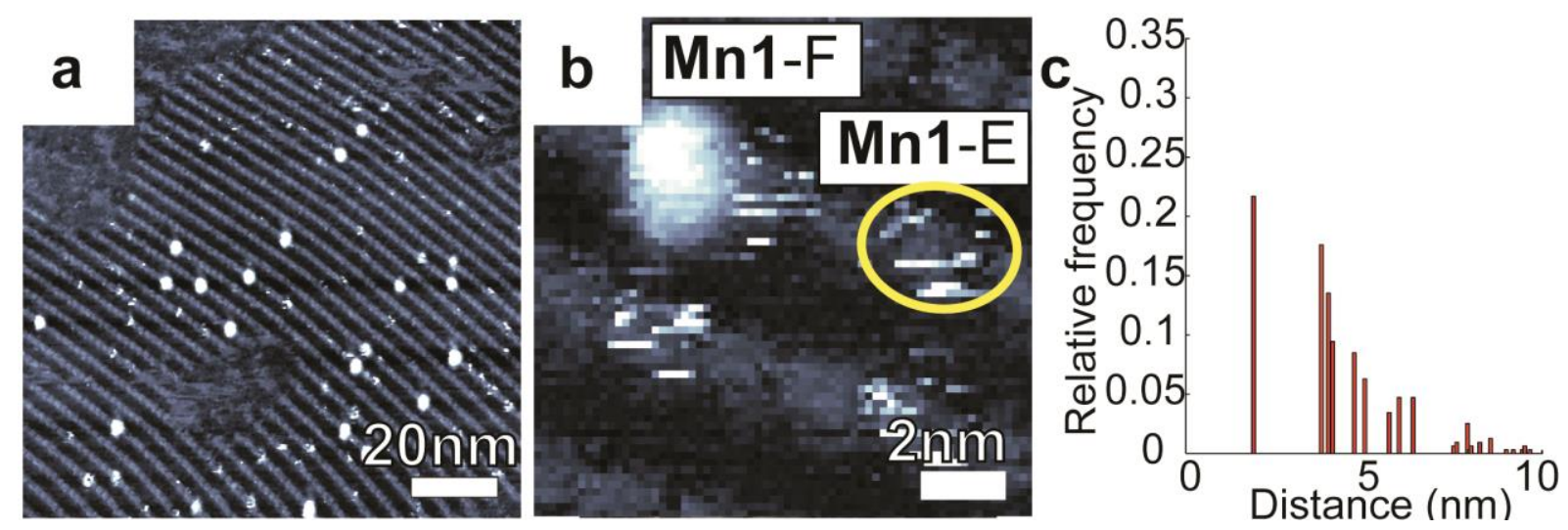

Figure 4 | Reaction of Mn-porphyrins with single oxygen donor PFIB at the graphite/1-octanoic acid interface. A, STM topography image 15 minutes after the addition of PFIB. $V_{\text {bias }}=-800 \mathrm{mV}$, Iset $=8 \mathrm{pA}$. B, Magnification, showing molecules of Mn1 in states Mn1-E (one of them included in the yellow ellipse) and Mn1-F after the addition of PFIB. C, Nearest neighbour distributions for Mn1-E in the STM experiment with PFIB, relative coverages between $10-16 \%(N=318, t=20 \min$ to $1.5 \mathrm{~h})$.

An Mn(IV)1-oxo complex can further react to a $\mu$-oxo bridged dimer by attaching to an additional Mn-porphyrin (Mn(III) ${ }^{+}$or $\left.\mathrm{Mn}(\mathrm{II})\right)$ from the supernatant solution or the surface. We propose such a dimer structure for state Mn1-D. Its appearance still features the "four-leaf clover" signature typically observed for a porphyrin plane (Fig. 5A), but oriented under an angle of approximately $45^{\circ}$ with respect to the other porphyrin planes in the same lamellar array that are directly adsorbed to the surface (Fig. 5B). The large apparent height of this complex of 0.4-0.5 nm, about twice the apparent height of Mn1-C, corresponds well to the topographical features of other "double decker"-like species studied by STM in which the two dye moieties are at similar distance ${ }^{38}$. The abundance of Mn1-D was dependent on the concentration of Mn1 in the supernatant solution and increased from $0.5 \%$ for $[\mathbf{M n 1}]=10^{-5}$ $\mathrm{M}$ to $3.4 \%$ for $[\mathbf{M n 1}]=10^{-4} \mathrm{M}$, after $4 \mathrm{~h}$ under ambient conditions. Since in an argon atmosphere Mn1-D was only sporadically observed after $4 \mathrm{~h}$ (never for $[\mathbf{M n 1}]=10^{-5} \mathrm{M}$ and $<0.5 \%$ abundance for $\left.[\mathbf{M n} 1]=10^{-4} \mathrm{M}\right)$, the presence of oxygen seemed to be a requirement for its formation. The concentration- and oxygen-dependence are in line with an assignment 
of Mn1-D as a $\mu$-oxo-bridged dimer. The fact that the top porphyrin is still quite wellresolved in the STM images indicates a hindered rotation along the Mn-O-Mn bond, imposed by steric interactions between the meso-phenyl groups of the two porphyrins as was also indicated by molecular modeling (Supplementary Fig. S14). Its concentration dependence indicates that the second porphyrin comes from the supernatant solution, in principle yielding a [Mn(III)1-O-Mn(IV)1 $]^{+}$dimer (see Fig. 1). Such a mixed-valence complex, however, has been reported unstable in solution ${ }^{25}$, and the observation that Mn1-D was generally stable for more than an hour suggests that such a dimer is either stabilised by its adsorption to the surface, or is reduced by a surface-induced reaction to a more stable $\mathbf{M n}$ (III)1-O-Mn(III)1 complex.
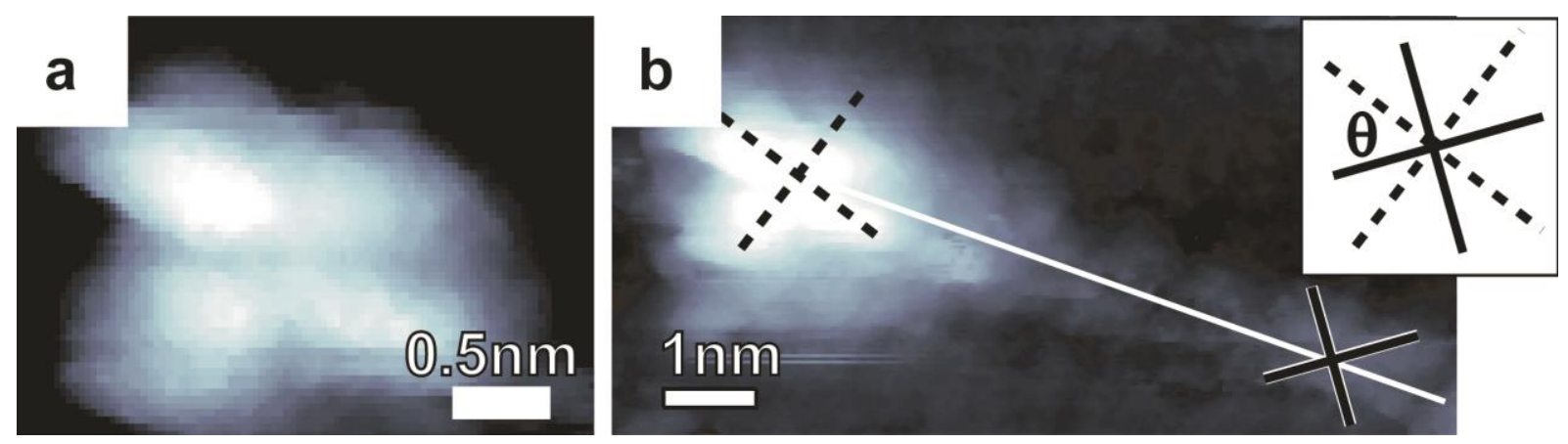

Figure 5 | Imaging of a $\mu$-oxo Mn-porphyrin dimer. A, High resolution STM topography of Mn1-D, revealing the submolecular structure of the porphyrin core; $V_{\text {bias }}=-800 \mathrm{mV}, I_{\text {set }}=10 \mathrm{pA}$. B, STM topography of an array of molecules of Mn1. The solid cross indicates the orientation of the porphyrin core of Mn1-A and the dotted cross that of the porphyrin core of Mn1-D. The white line connects the centres of the molecules. Inset: indication of the angle $\theta=45 \pm 5^{\circ}$ between the two orientations; $V_{\text {bias }}=-800 \mathrm{mV}$, $I_{\text {set }}=10 \mathrm{pA}$.

While Mn1-F (Figs 4A and 4B) has a very similar appearance and apparent height as Mn1-D, the expected $\mu$-oxo-bridged dimer formed from a $[\mathbf{M n}(\mathbf{V}) \mathbf{1 - o x o}]^{+}$species would be a $[\mathrm{Mn}(\mathbf{I V}) 1-\mathrm{O}-\mathrm{Mn}(\mathbf{I V}) \mathbf{1}]^{2+}$ complex. The STM topographies are not conclusive as to whether or not Mn1-F is identical to Mn1-D (for example via a surface-induced reduction), or in fact a slightly different state with a similar topographical appearance.

Mn1-C and Mn1-D are both proposed to be generated after a reaction of Mn1 with $\mathrm{O}_{2}$. Before a manganese porphyrin can bind and split oxygen, its manganese centre needs to be reduced from $\mathrm{Mn}(\mathrm{III})$ to $\mathrm{Mn}(\mathrm{II})^{1}$. Since $\mathrm{Mn1}$ in solution is inert towards oxygen, we propose 
that in our STM experiments Mn1 is reduced by the negatively biased HOPG surface ${ }^{39,40}$, and that the hitherto unassigned state Mn1-B corresponds to an Mn(II) species. Several arguments, which will be discussed in the following, are in support of this assignment.

The emergence of Mn1-B, Mn1-C and Mn1-D appeared to be strongly dependent on the applied bias voltage. At positive sample bias voltages and low tunneling current setpoints, the monolayer of Mn1 is unstable and typically desorbs within 30 minutes. At a bias voltage of $-500 \mathrm{mV}$ under ambient conditions and an $\mathrm{I}_{\text {set }}$ of $5 \mathrm{pA}$ or lower, Mn1-B was never observed, no more than $1 \%$ of Mn1-C was present after 4 h, and Mn1-D was observed only occasionally. Under the same conditions but at $-800 \mathrm{mV}$, these relative coverages had increased to approximately $15 \%, 5 \%$ and $0.3 \%$, respectively (Fig. 6A). We propose that a sufficiently negative bias on the surface is required to reduce Mn1-A to Mn1-B, an Mn(II) porphyrin, and attribute the observation that not every manganese(III) porphyrin in the monolayer is reduced to a non-ideal coupling between the porphyrin plane and the graphite surface, imposed by the meso-phenyl substituents. Although these substituents can freely rotate, a full alignment of their aromatic planes with the porphyrin plane is highly unfavorable because of steric hindrance with the hydrogen atoms attached to the pyrrole rings. As a result, it is difficult for the manganese atom to come in close contact with the surface, which will decrease the probability of the donation of an electron from the surface (see also the Supplementary Note). Previous research on a monolayer of Mn(III) porphyrins that lacked meso-phenyl substituents revealed that their adsorption to a $\mathrm{Au}(111)$ surface led to a quantitative reduction of the manganese centres from $\mathrm{Mn}$ (III) to $\mathrm{Mn}(\mathrm{II})^{18}$. While a difference in work function between the two surfaces might play a role in this process, it was reasoned that in the case of $\mathrm{Au}(111)$ a direct contact between the surface and the metal centre was possible, and that gold adatoms could act as axial ligands to the porphyrin metals ${ }^{41,42}$. Axial ligand effects between a surface and adsorbed molecules have been studied comprehensively between a $\mathrm{Ag}(111)$ surface and various metal-porphyrins in UHV, and it was shown that such interactions can have considerable influence on the electronic state of the porphyrins and their reactivity. ${ }^{44}$ But also in the absence of a direct chemical bond a surface like graphite can activate a metal centre, as was demonstrated in a recent STM study of the oxygenation of cobalt porphyrins at the graphite/1-phenyloctane interface. ${ }^{19}$ In that case the graphite surface was proposed to stabilise the polarised porphyrin $\mathrm{Co}-\mathrm{O}_{2}$ complex by means of charge donation. 
Upon its formation, Mn1-B becomes an active site for molecular oxygen and can act as a precursor of the oxygen-containing species Mn1-C and Mn1-D. This precursor role was supported by several dynamic phenomena observed by STM. In subsequent STM images recorded at time intervals of 2.5 minutes, Mn1-B appeared to be 4 times more likely to transform into Mn1-C than molecules of Mn1-A into Mn1-C (Supplementary Table S1). In one occasion, a molecule of Mn1-A was observed to switch to Mn1-B in between two scan lines, and in the next STM image to Mn1-C (Fig. 6B).

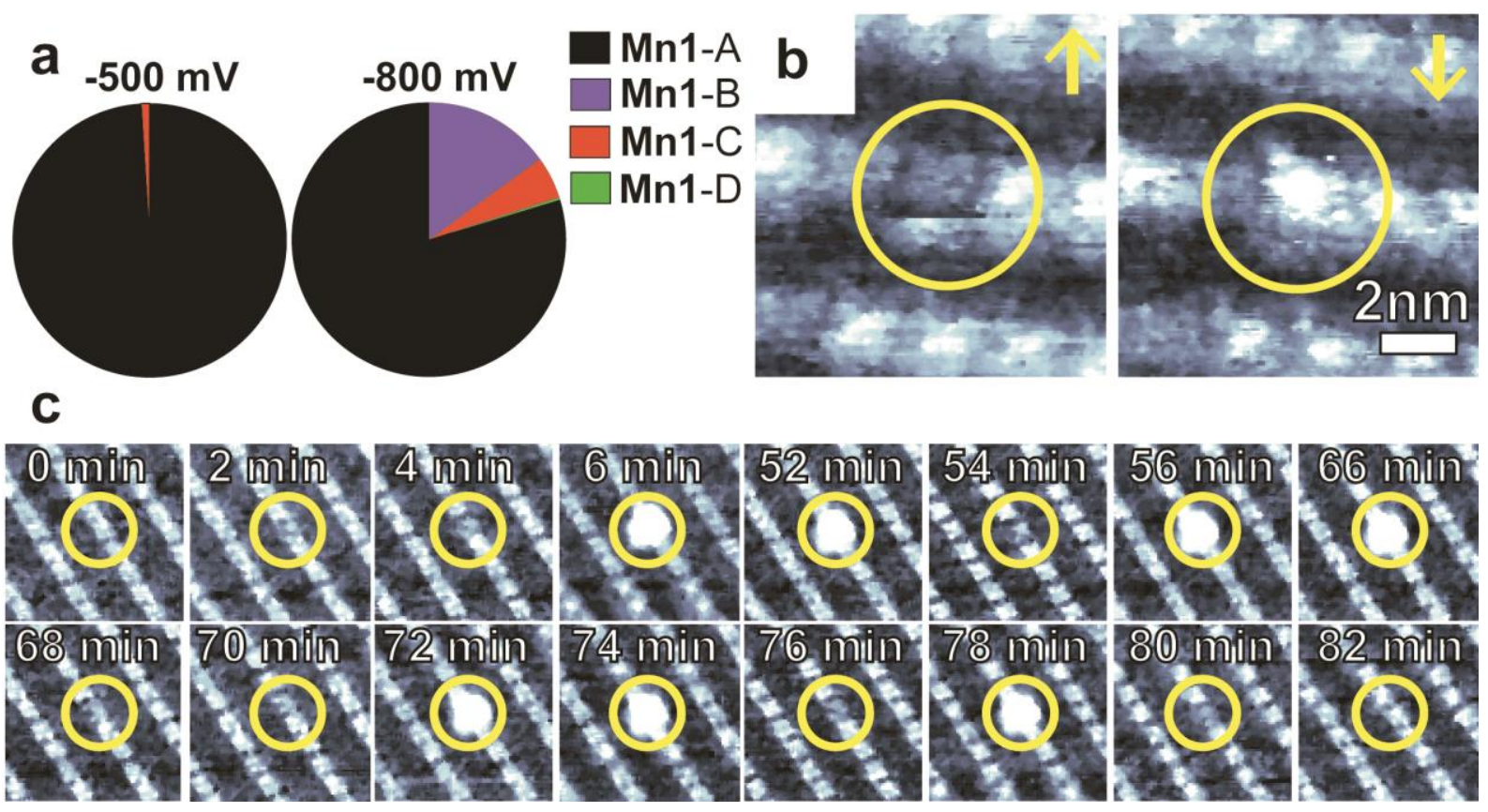

Figure 6 | Imaging of a reactive Mn-porphyrin state. A, Distribution of states of Mn1 after $4 \mathrm{~h}$ under ambient conditions, at $V_{\text {bias }}=-500 \mathrm{mV}$ and $-800 \mathrm{mV} ; \mathrm{I}_{\text {set }}=5 \mathrm{pA}$. B, A single molecule of Mn1, which converts from Mn1-A to Mn1-B in between two scan lines (time interval $380 \mathrm{~ms}$ ), and subsequently to $\mathbf{M n 1 - C}$ in the next image (time interval 2 min.). The arrows in the top right corners of the images indicate the scan direction. C, Series of sequential STM topographies in which a molecule of Mn1 (in the yellow circle) switches between various states; $V_{\text {bias }}=-800 \mathrm{mV}, I_{\text {set }}=10 \mathrm{pA}$.

The reactive nature of Mn1-B is further illustrated in Fig. 6C, where a sequence of STM images in one experiment is shown. A molecule of Mn1-B converts to Mn1-D, which remains stable for almost an hour, and then starts to switch repeatedly between Mn1-D and Mn1-B, until Mn1-B returns to state Mn1-A and becomes unreactive (see also the Supplementary Video). The observed series of frequent and reversible transitions at the same location over such a long period of time is highly remarkable, and not straightforward to 
explain. They can be attributed to a repetitive formation and decomposition of an Mn(III)1O-Mn(III)1 complex at an Mn(II)1 active site which remains immobilised at the surface. The dynamic particle involved is proposed to be an $\mathbf{M n}(\mathbf{I V}) \mathbf{1 - o x o}$ species, which apparently resides nearby the active site, either in solution, connected via van der Waals interactions to nearby molecules in the monolayer, or attached to the STM tip.

This work has shown that it is possible to discriminate different states of a reactive species with STM and thereby to monitor complex multistep reactions at the submolecular level at room temperature and under different atmospheric conditions. The dynamic and chemically realistic environment of a solid/liquid interface allows the direct observation of transitions between single reactants, intermediates and products, which provides unique mechanistic details and reaction rates that remain obscured when a reaction is studied by an ensemble technique. Of particular interest is the possibility to use a surface in the role of cofactor to deliver electrons to a reaction, which in principle allows for control of the reactivity of adsorbed molecules. The exploration of surface-mediated reactivity at the single molecule level with scanning tunneling microscopy under environmental control may lead to the discovery of new reactions and reaction pathways. The nanoscale probe itself might also be used as a tool to control surface-mediated reactivity on demand with (sub)molecular precision.

\section{Methods}

The STM measurements were performed in the constant current mode with an environmentally controlled home-built low-current STM, using an Omicron Scala SPM controller. STM tips were mechanically cut from Pt/Ir wires (90:10) with a diameter of 0.5 $\mathrm{mm}$. The highly oriented pyrolytic graphite used was grade ZYB (NTMDT, Zelenograd, Moscow). STM measurements were performed at the graphite/solution interface, with the tip immersed in a droplet of the solution of the molecules. The sizes of the pieces of graphite were in the range of $2-8 \mathrm{~mm}^{2}$, and the volume of the applied droplets between $3-5 \mu$. The unit cell of the molecular layer was determined by imaging the underlying graphite during the measurements of the molecules by changing the feedback parameters ( $V_{\text {bias }}$ and $I_{\text {set }}$ ), and subsequently the graphite lattice dimensions were used for calibration. All STM measurements shown have been corrected for the background, and a $3 \times 3$ median filter was applied. The solutions of Mn1 in 1-octanoic acid (Sigma-Aldrich, distilled before use) were 
typically $2-3 \times 10^{-5} \mathrm{M}$ in concentration, unless otherwise indicated in the text. Prior to the experiments the solution was argon purged for at least a night and often several weeks, to remove dissolved oxygen. In order to make a reliable comparison between the measurements carried out in oxygen-poor and oxygen-rich atmospheres, special care was taken that, besides the concentration of oxygen, the other factors were kept as similar as possible: the experiments were performed with the same batch of solution, only one day apart, after argonpurging for two weeks. $V_{\text {bias }}$ and $I_{\text {set }}$ were kept exactly the same in both experiments. The experiments in which the effect of $V_{\text {bias }}$ was studied were performed with the same solution, in ambient conditions after the solution was argon-purged overnight, and with the same $I_{\text {set. }}$.

\section{References and Notes}

1. Tabushi, I. Reductive dioxygen activation by use of artificial cytochrome P-40 systems. Coord. Chem. Rev., 86, 1-42 (1988).

2. Feiters, M. C., Rowan, A. E. \& Nolte, R. J. M. From simple to supramolecular cytochrome P450 mimics. Chem. Soc. Rev., 29, 375-384 (2000).

3. Meunier, B. Metalloporphyrins as versatile catalysts for oxidation reactions and oxidative DNA cleavage. Chem. Rev., 92, 1411-1456 (1992).

4. Mansuy, D. Activation of alkanes - the biomimetic approach. Coord. Chem. Rev., 125, 129-141 (1993).

5. Groves, J. T. In: Cytochrome P450: Structure, Mechanism, and Biochemistry, 3rd ed.; Ortiz de Montellano, P. R., Ed.; Kluwer Academic/Plenum Publishers: New York, 2005; pp 1-43.

6. Song, W. J. et al. Synthesis, characterization, and reactivities of manganese(V)-oxo porphyrin complexes. J. Am. Chem. Soc., 129, 1268-1277 (2007).

7. Zhang, R., Horner, J. H. \& Newcomb, M., Laser flash photolysis generation and kinetic studies of porphyrin-manganese-oxo intermediates. Rate constants for oxidations effected by porphyrin-Mn-V-oxo species and apparent disproportionation equilibrium constants for porphyrin-Mn-IV-oxo species. J. Am. Chem. Soc., 127, 6573-6582 (2005). 
8. Grim, P. C. M. et al., Submolecularly resolved polymerization of diacetylene molecules on the graphite surface observed with scanning tunneling microscopy. Angew. Chem. Int. Ed., 36, 2601-2603 (1997).

9. Hla, S.-W., Bartels, L., Meyer, G. \& Rieder, K. H., Inducing all steps of a chemical reaction with the scanning tunneling microscope tip: Towards single molecule engineering. Phys. Rev. Lett., 85, 2777-2780 (2000).

10. Okawa, Y. \& Aono, M., Nanoscale control of chain polymerization Nature, 409, 683-684 (2001).

11. Piot, L., Bonifazi, D. \& Samorì, P. Organic reactivity in confined spaces under scanning tunneling microscopy control: Tailoring the nanoscale world. Adv. Funct. Mater., 17, 36893693 (2007).

12. Grill, L. et al., Nano-architectures by covalent assembly of molecular building blocks. Nat. Nanotechnol., 2, 687-691 (2007).

13. Turner, M. et al., Deprotection, tethering, and activation of a catalytically active metalloporphyrin to a chemically active metal surface: [SAc](4)P-Mn(III)Cl on $\mathrm{Ag}(100) . J$. Am. Chem. Soc., 131, 1910-1914 (2009).

14. Turner, M. et al., Deprotection, tethering, and activation of a one-legged metalloporphyrin on a chemically active metal surface: NEXAFS, synchrotron XPS, and STM study of [SAc]PMn(III)Cl on Ag(100). J. Am. Chem. Soc., 131, 14913-14919 (2009).

15. Seufert, K., Auwärter, W. \& Barth, J. V. Discriminative response of surface-confined metalloporphyrin molecules to carbon and nitrogen monoxide. J. Am. Chem. Soc., 132, 18141 (2010).

16. Beggan, J. P. et al., Control of the axial coordination of a surface-confined manganese(III) porphyrin complex. Nanotechnology, 23, 235606 (2012).

17. Gopakumar, T. G., Tang, H., Morillo, J. \& Berndt, R. Transfer of Cl ligands between adsorbed iron tetraphenylporphyrin molecules. J. Am. Chem. Soc., 134, 11944 (2012).

18. Hulsken, B. et al., Real-time single-molecule imaging of oxidation catalysis at a liquidsolid interface. Nat. Nanotechnol., 2, 285 (2007). 
19. Friesen, B. A., Bhattarai, A., Mazur, U. \& Hipps, K. W. Single molecule imaging of oxygenation of cobalt octaethylporphyrin at the solution/solid interface: thermodynamics from microscopy. J. Am. Chem. Soc., 134, 14897-14904 (2012).

20. Reed, C. A., Kouba, J. K., Grimes, C. J. \& Cheung, S. K. Manganese(II) and chromium(II) porphyrin complexes - synthesis and characterization. Inorg. Chem., 17, 26662670 (1978).

21. Creager, S. E., Raybuck, S. A. \& Murray, R. W. An efficient electrocatalytic model cytochrome-P-450 epoxidation cycle. J. Am. Chem. Soc., 108, 4225-4227 (1986).

22. Lyons, J. E., Ellis, P. E. \& Myers, H. K. Halogenated metalloporphyrin complexes as catalysts for selective reactions of acyclic alkanes with molecular oxygen. J. Catal., 155, 5973 (1995).

23. Jones, R. D., Summerville, D. A. \& Basolo, F. Synthetic oxygen carriers related to biological systems. Chem. Rev., 79, 139-179 (1979).

24. Watanabe, Y. \& Fujii, H. in: Metal-oxo and metal-peroxo species in catalytic oxidations, pp. 61 - 89, Springer, Berlin, 2000.

25. Schardt, B. C., Hollander F. J. \& Hill, C. L. Isolation, purification, and characterization of high-valent complexes from a manganese porphyrin based catalytic hydrocarbon activation system - crystal and molecular-structure of mu-oxo-bis[azido(tetraphenylporphinato)manganese(IV)]. J. Am. Chem. Soc., 104, 3964-3972 (1982).

26. Smegal, J. A., Schardt, B. C. \& Hill, C. L. Isolation, purification, and characterization of intermediate (iodosylbenzene)metalloporphyrin complexes from the (tetraphenylporphinato)manganese(III)-iodosylbenzene catalytic hydrocarbon functionalization system. J. Am. Chem. Soc., 105, 3510-3515 (1983).

27. Nolte, R. J. M., Razenberg J. A. S. J. \& Schuurman, R. On the rate-determining step in the epoxidation of olefins by monooxygenase models. J. Am. Chem. Soc., 108, 2751-2752 (1986). 28. Van der Made, A. W., Nolte, R. J. M. \& Drenth, W. On the mechanism of epoxidation of alkenes with hypochlorite, catalyzed by manganese(III) tetraarylporphyrins. Recl. Trav. Chim. Pays-Bas, 109, 537-551 (1990). 
29. Dismukes, G. C. Sheats J. E. \& Smegal, J. A. $\mathrm{Mn}^{2+} / \mathrm{Mn}^{3+}$ and $\mathrm{Mn}^{3+} / \mathrm{Mn}^{4+}$ mixed-valence binuclear manganese complexes of biological interest. J. Am. Chem. Soc., 109, 7202-7203 (1987).

30. Linares, M. et al. Chiral expression at the solid-liquid interface: A joint experimental and theoretical study of the self-assembly of chiral porphyrins on graphite. Langmuir 24, 95669574 (2008).

31. Iavicoli, P. et al., Tuning the supramolecular chirality of one- and two-dimensional aggregates with the number of stereogenic centers in the component porphyrins J. Am. Chem. Soc. 132, 9350-9362 (2010).

32. Hoffman, B. M., Weschler, C. J. \& Basolo, F. Dioxygen adduct of mesotetraphenylporphyrinmanganese(II), a synthetic oxygen carrier. J. Am. Chem. Soc. 98, 54735482 (1976).

33. Hoffman, B. M., Szymanski, T., Brown, T. G. \& Basolo, F. Dioxygen adducts of several manganese(II) porphyrins - electron-paramagnetic resonance studies. J. Am. Chem. Soc., 100, 7253-7259 (1978).

34. Jones, R. D., Summerville, D. A. \& Basolo, F. Manganese(II) porphyrin oxygen carriers equilibrium-constants for reaction of dioxygen with para-substituted mesotetraphenylporphinatomanganese(II) complexes. J. Am. Chem. Soc. 100, 4416 (1978).

35. Jones, R. D., Summerville, D. A. \& Basolo, F. Synthetic oxygen carriers related to biological-systems. Chem. Rev. 79, 139-179 (1979).

36. Fabris, S. et al., Oxygen dissociation by concerted action of di-iron centers in metalorganic coordination networks at surfaces: modeling non-heme iron enzymes. Nano Lett., 11, 5414-5420 (2011).

37. Huskens, J. et al., A model for describing the thermodynamics of multivalent host-guest interactions at interfaces. J. Am. Chem. Soc. 126, 6784-6797 (2004). 38. Miyake, K. et al., Molecular motion of surface-immobilized double-decker phthalocyanine complexes. J. Am. Chem. Soc., 131, 17808-17813 (2009). 
39. Oyaizu, K., Haryono, A., Yonemaru, H. \& Tsuchida, E. Catalytic behavior of a mu-oxo dimanganese(III) octaethylporphyrin in O-2 reduction. J. Chem. Soc., Faraday Trans., 94, 3393-3399 (1998).

40. He, Y. \& Borguet, E. Dynamics of porphyrin electron-transfer reactions at the electrodeelectrolyte interface at the molecular level. Angew. Chem. Int. Ed., 46, 6098-6101 (2007).

41. Bai, Y. et al., Interfacial coordination interactions studied on cobalt octaethylporphyrin and cobalt tetraphenylporphyrin monolayers on $\mathrm{Au}(111)$. Phys. Chem. Chem. Phys., 12, 4336 (2010).

42. Suárez, S. A. et al., A Surface Effect Allows HNO/NO Discrimination by a Cobalt Porphyrin Bound to Gold. Inorg. Chem., 49, 6955-6966 (2010).

43. Hieringer, W. et al., The surface trans effect: influence of axial ligands on the surface chemical bonds of adsorbed metalloporphyrins. J. Am. Chem. Soc. 133, 6206-6222 (2011).

\section{Acknowledgements}

NanoNed (the Dutch nanotechnology initiative by the Ministry of Economic Affairs), the Foundation for Fundamental Research on Matter (FOM programme Atomic and Molecular Nanophysics), and the Ministry of Education, Culture and Science (Gravity program 024.001.035) are acknowledged for financial support. D.B.A. acknowledges funding for projects CTQ2010-16339) and 2009 SGR 158. S.D.F. thanks the Fund of Scientific Research-Flanders (FWO), KU Leuven (GOA), and the Belgian Federal Science Policy Office (IAP-P7/05). J.A.A.W.E. and T.H. thank the Council for the Chemical Sciences of The Netherlands Organisation for Scientific Research, CW-NWO, for a Vidi grant (700.58.423) and an ECHO grant (700.57.023), respectively. J.A.A.W.E. thanks the European Research Council for an ERC Starting Grant (NANOCAT - 259064).

\section{Author contributions}

J.A.A.W.E., S.D.F. and D.B.A. conceived the idea and designed the experiments. D.d.B. and M.L. performed the STM experiments. T.H. and D.d.B. carried out data analyses and P.I. 
synthesised and characterised the compound. D.d.B, M.L. and J.A.A.W. co-wrote the paper. All authors analysed and discussed the results and commented on the manuscript.

\section{Additional information}

Supplementary information and chemical compound information accompany this paper at www.nature.com/naturechemistry. Reprints and permission information is available online at http://www.nature.com/reprints. Correspondence and requests for materials should be addressed to J.A.A.W.E., S.D.F., and D.B.A.

\section{Competing financial interests}

The authors declare no competing financial interests. 